\title{
Targeted Therapy of Colon Cancer by Aptamer-Guided Holliday Junctions Loaded with Doxorubicin
}

This article was published in the following Dove Press journal: International Journal of Nanomedicine

\section{Fengjiao Yao \\ Yacong An \\ Xundou Li \\ Zhaoyi Li \\ Jinhong Duan \\ Xian-Da Yang}

Institute of Basic Medical Sciences, Chinese Academy of Medical Sciences and Peking Union Medical College, Beijing, People's Republic of China
Correspondence: Xian-Da Yang Email ayangmd@hotmail.com
Purpose: Chemotherapy is the primary treatment for advanced colon cancer, but its efficacy is often limited by severe toxicities. Targeted therapy in the form of selectively drug delivery system (SDDS) is an important strategy to reduce adverse effects. Here, we aim to design a novel SDDS with potential for practical application using biocompatible components and scalable production process, for targeted delivery of doxorubicin (Dox) to colon cancer cells. Methods: The SDDS was made of a self-assembled DNA nano-cross (Holliday junction, or HJ) functionalized by four AS1411 aptamers (Apt-HJ) and loaded with Dox.

Results: Apt-HJ had an average size of $12.45 \mathrm{~nm}$ and a zeta potential of $-11.6 \mathrm{mV}$. Compared with the monovalent AS1411 aptamer, the quadrivalent Apt-HJ showed stronger binding to target cancer cells (CT26). A complex of Apt-HJ and doxorubicin (Apt-HJ-Dox) was formed by intercalating Dox into the DNA structure of Apt-HJ, with each complex carrying approximately 17 Dox molecules. Confocal microscopy revealed that Apt-HJ-Dox selectively delivered Dox into CT26 colon cancer cells but not the control cells. Moreover, Apt-HJ-Dox achieved targeted killing of CT26 cancer cells in vitro and reduced the damage to control cells. Importantly, compared with free Dox, Apt-HJ-Dox significantly enhanced the antitumor efficacy in vivo without boosting the adverse effects.

Conclusion: These results suggest that Apt-HJ-Dox has application potential in targeted treatment of colon cancer.

Keywords: colon cancer, targeted therapy, aptamer, holliday junction, doxorubicin

\section{Introduction}

Colon cancer is a severe threat to human health, with incidence and mortality ranking the fourth and the fifth among all cancers, respectively. It is estimated that there were approximately 1.1 million new colon cancer cases and 0.6 million related deaths in 2018 worldwide. ${ }^{1}$ In recent years, the incidence of colon cancer has been increasing, especially for adolescents and young adults. ${ }^{2}$ With the development of world economy and the changes in lifestyle and diet, the incidence of colon cancer is expected to further increase. ${ }^{3}$ For early-stage colon cancer, surgery is the treatment of choice. For advanced colon cancer, however, chemotherapy is still the primary treatment modality, because drugs have systemic distribution and can reach metastatic cancer cells theoretically. ${ }^{4,5}$ One of the main problems of chemotherapy is the severe side effects associated with cytotoxic agents, including nausea, vomiting, diarrhea, weight loss, abdominal pain, leucopenia, etc. ${ }^{6,7}$ These adverse effects significantly limit treatment duration and drug dosage, therefore 
compromising the therapeutic efficacy. Hence, reducing drug-related side effects is critical for further improving the clinical outcome of chemotherapy.

A promising strategy to reduce adverse effects is targeted drug delivery to cancer cells. This approach is often implemented with selective drug delivery system (SDDS), which typically consists of three components: anticancer drugs, drug carrier in the form of nanoparticle or nanostructure, and tumor-targeting ligand conjugated to the carrier. When tumor-targeting ligand binds with cancer cells, the drug-carrier is endocytosed and releases the anticancer drugs. Because SDDS can target cancer cells but not healthy tissues, therapeutic efficacy is enhanced and offtarget toxicity is reduced. Previous studies have shown that targeted drug delivery may potentially improve the treatment outcome. Yu et al designed a MUC1 aptamerguided nanoscale drug delivery system and demonstrated that the system could effectively enhance the paclitaxel delivery to MUC1-expressing breast cancer cells. ${ }^{8}$ Zhang et al designed a biotin and hyaluronidase dual-responsive SDDS (MSN-HA/Dox) and found that it triggered enhanced apoptosis among cancer cells in vitro and conferred better antitumor effects in vivo. ${ }^{9}$ Recently, several targeted cancer therapies in the form of antibody-drug conjugates (ADC) have been approved by the FDA for clinical applications. Brentuximab vedotin (Adcetris ${ }^{\circledR}$ ) was approved for relapsed Hodgkin lymphoma and systemic anaplastic large cell lymphoma, trastuzumab emtansine $\left(\right.$ Kadcyla $\left.^{\circledR}\right)$ for HER2-positive metastatic breast cancer, and inotuzumab ozogamicin (Besponsa ${ }^{\circledR}$ ) for acute lymphoblastic leukemia. ${ }^{10,11}$ In addition, there are over 60 ADCs clinical trials ongoing currently. ${ }^{12}$ These developments indicate that targeted delivery of anticancer drug is an important trend for future chemotherapy.

In order to build an SDDS for targeted drug delivery to cancer tissue, tumor-homing ligands are required. Antibody is frequently employed as the ligand because of its high affinity and specificity. In addition to antibodies, aptamers can also be used as novel tumor-targeting ligands. Aptamers are short, single-stranded oligonucleotides (DNA or RNA) that can form unique three-dimensional structures to serve as ligands that bind with the target molecules. Aptamers can not only bind to the targets with high affinity and specificity but have better penetration of tumor tissues due to small molecular size. ${ }^{13}$ What's more, aptamers can be chemically synthesized at low cost and easily modified with various functional groups. ${ }^{13}$ Moreover, aptamer-based drug has been approved by FDA for clinical application: Macugen was approved for treating age-related wet macular degeneration. ${ }^{14}$ Aptamers, therefore, have potential in the development of targeted therapeutics for clinical applications.

A typical SDDS has drug-carrier for transporting cytotoxic agents to cancer cells. Most drug carriers are nanoparticles made of organic or polymeric molecules. ${ }^{15}$ In addition to these nanoparticles, DNA nanostructure can also serve as an excellent drug carrier. DNA nanostructure can carry doxorubicin, which tends to intercalate into double-stranded DNA within the structure. ${ }^{16}$ Doxorubicin is a broad spectrum anticancer therapeutics that can be used to treat a variety of malignancies. DNA nanostructure has negligible immunogenicity with good biocompatibility, and can be easily fabricated in a self-assembled manner. Holliday junction (HJ) is a DNA nanostructure with promising drug carrier potential. It is made of four single-strand DNA chains locked into the shape of a cross. ${ }^{17}$ The size of HJ is relatively small, with a mean size of about $10 \mathrm{~nm}$. Because prior studies have shown that smaller nanoparticles can penetrate tumor and lymph nodes more efficiently vs. larger nanoparticles. ${ }^{18}$ The small size of HJ may allow it to enter cancer tissues and lymph nodes more efficiently and serve as a better drug carrier of doxorubicin towards lymph nodes, which often host metastatic cancer cells. ${ }^{19}$ Moreover, HJ has four open ends that can be linked to four tumor-targeting aptamers, thereby improving its affinity to target cancer cells.

To date, however, aptamer-modified $\mathrm{HJ}$ has not been used as a drug carrier of Dox to treat colon cancer. In this study, we designed and fabricated a novel SDDS consisting of an HJ modified by four AS1411 aptamers, for targeted delivery of Dox to colon cancer cells. AS1411 is a DNA aptamer that can recognize and bind with nucleolin, which is a protein overexpressed on the cell membrane of multiple malignancies, including colon cancer. $^{20-22}$ The results indicated that AS1411-modified HJ could selectively deliver doxorubicin to murine colon cancer cells in vitro, and generated stronger antitumor efficacy in vivo compared with free Dox.

\section{Materials and Methods Synthesis of DNAs}

All DNAs were synthesized by Invitrogen (Shanghai, China). The sequence used in this study is shown in Table 1.

\section{Cell Culture}

Mouse colon cancer cell line (CT26) and Chinese hamster ovary cell line (CHO) were purchased from the Cell Center of Chinese Academy of Medical Sciences 
Table I The DNA Sequence Used in This Study

\begin{tabular}{|l|l|}
\hline Oligonucleotide & Sequence (from $\mathbf{5}$ ' to $\mathbf{3}^{\prime}$ ) \\
\hline Sequence 1 & CGGCGATCCGGCCATAGTGGATTGCGGGCCAGTGAAAAAAGGTGGTGGTGGTTGTGGTGGTGGTGG \\
Sequence 2 & CTCACTGGCCCGCAATCCTGAGCACGTGGCTGACGAAAAAGTGGTGGTGGTTGTGGTGGTGGTGG \\
Sequence 3 & CCGTCAGCCACGTGCTCACCGAATGCTGCGCAACCAAAAAGGTGTGGTGGTTGTGGTGGTGGTGG \\
Sequence 4 & CGGTTGCGCAGCATTCGGACTATGGCCGGATCGCCAAAAAGGTGGTGGTGGTTGTGGTGGTGGTGG \\
\hline
\end{tabular}

Notes: The underlined parts indicated the sequence of ASI4II aptamer.

(Beijing, China). Cells were cultured in DMEM medium supplemented with $10 \%$ fetal bovine serum (FBS) and $1 \%$ penicillin/streptomycin (penicillin: 100 units/mL, streptomycin: $100 \mathrm{mg} / \mathrm{mL}$ ). All cells were incubated at $37^{\circ} \mathrm{C}$ in incubator with 5\% CO2 and 95\% air. The cell culture medium was purchased from the Cell Center of Chinese Academy of Medical Sciences (Beijing, China). FBS was purchased from BioInd (Israel).

\section{Preparation of Apt-HJ}

Four single-strand DNAs were mixed in saline solution $(0.9 \% \mathrm{NaCl}, 10 \mathrm{mM} \mathrm{MgCl} 2)$ at an equal molar ratio. The mixture was incubated at $95^{\circ} \mathrm{C}$ for $5 \mathrm{mins}$, and then slowly cooled to the room temperature at the rate of $0.1^{\circ} \mathrm{C} / \mathrm{s}$.

\section{Characterization of Apt-HJ}

Agarose gel electrophoresis was employed to validate the formation of Apt-HJ. The gel was prepared by TBE buffer with $2.5 \%(\mathrm{w} / \mathrm{v})$ agarose and GelStain (TransGen Biotech, Beijing, China). The gel was run at $110 \mathrm{~V}$ for $20 \mathrm{~min}$ analyzed under UV light. The size and zeta-potential of Apt-HJ were determined by dynamic light scattering (Malvern Zetasizer Nano ZS, UK).

\section{Serum Stability of Apt-HJ}

Phosphorothioate-modified and non-modified Apt-HJs were prepared at the concentration of $4 \mu \mathrm{M}$. DNA samples were incubated with $50 \%$ fetal bovine serum (FBS) at $37^{\circ}$ C. After various degradation time, samples were collected and heated at $95^{\circ} \mathrm{C}$ for 5 min immediately. $2.5 \%$ agarose gel electrophoresis was employed to detect the degradation of the DNA nanostructures. Quantity one software was used to detect the gray-scale value.

\section{Cellular Binding Assays}

Flow cytometry was performed to compare the cell-binding capability of monovalent aptamer with Apt-HJ. CT26 and $\mathrm{CHO}$ cells were incubated in PBS (plus $0.02 \%$ EDTA) for $30 \mathrm{~s}$ and washed with PBS twice gently. The cells $\left(2 \times 10^{5}\right)$ were suspended in $250 \mu \mathrm{L}$ of PBS, incubated with equimolar (60 pmol/tube) FAM-labeled random DNA, Apt, Apt-HJ separately for 30 mins with gentle shaking, washed twice with PBS, and resuspended in $250 \mu \mathrm{L}$ PBS. The samples were analyzed by flow cytometry (Accuri C6, BD, San Jose, CA, USA). FlowJo 7.6 software was used to analyze the data. Experiments for binding assays were repeated three times.

\section{Drug-Loading Capacity of Apt-HJ}

Apt-HJ was incubated with fixed concentration of Dox ( $3 \mathrm{nM})$ for $1 \mathrm{~h}$ in a 96-well black plate at designated carrier/Dox molar ratios. The fluorescence spectrum of Dox was examined by a Synergy4 analyzer $(\lambda \mathrm{Ex}=480 \mathrm{~nm}, \lambda \mathrm{Em}=520-720 \mathrm{~nm})$.

\section{Preparation of Apt-HJ-Dox}

To prepare Apt-HJ-Dox, Apt-HJ was assembled first. Four single-strand DNAs were mixed in saline solution $(0.9 \%$ $\mathrm{NaCl}, 10 \mathrm{mM} \mathrm{MgCl} 2)$ at an equal molar ratio. The mixture was incubated at $95^{\circ} \mathrm{C}$ for $5 \mathrm{mins}$, and slowly cooled to the room temperature at the rate of $0.1{ }^{\circ} \mathrm{C} / \mathrm{s}$ in a PCR machine. Subsequently, Apt-HJ was mixed with Dox at the molar ratio of $1: 17$. The mixture was incubated at $37^{\circ} \mathrm{C}$ for $1 \mathrm{hr}$, to form the Apt-HJ-Dox complex.

\section{Confocal Fluorescence Microscopy Imaging}

To study the cellular uptake of Dox, confocal imaging assay was performed. CT26 and CHO cells $\left(1.5 \times 10^{4}\right)$ were cultivated in Lab-Tek Chamber \#1.0 Borosilicate Coverglass System (ThermoFisher, Waltham, MA, USA) for $12 \mathrm{~h}$. The cells were incubated with free Dox and AptHJ-Dox at an equivalent dose of Dox $(2 \mu \mathrm{M})$ for $1 \mathrm{~h}$ and washed three times with PBS. The cells were fixed with $4 \%$ paraformaldehyde solution for 10 mins at $4{ }^{\circ} \mathrm{C}$, which was freshly prepared and pre-cooling. The cells were washed thrice with PBS and dyed with Hoechst (Solarbio, Beijing, China) for $30 \mathrm{~min}$ at room temperature. After being washed for three times, the cells were incubated with PBS and evaluated with confocal fluorescence microscopy (UltraVIEW VOX). 


\section{Cytotoxicity Assays in vitro}

Cells were seeded on 96-well plate at a concentration of 5000 cells per well. After overnight cultivation, the cells were treated with Apt-HJ, free Dox or Apt-HJ-Dox at an equivalent dose of Dox (CT26: $3 \mu \mathrm{M}$; CHO: $2 \mu \mathrm{M}$ ) for $1 \mathrm{~h}$ at $37^{\circ} \mathrm{C}$. The cells were washed twice with PBS and cultured for additional $48 \mathrm{hrs}$. MTS assay (Promega, Madison, WI, USA) was used to determine the cell viability per the standard protocol suggested by the manufacture. The experiment was repeated three times.

\section{Evaluation of IC50}

Cells were cultured in 96-well plate as described before and incubated with free Dox or Apt-HJ-Dox for $1 \mathrm{~h}$ at $37^{\circ}$ C. CT26 cells were treated with equivalent Dox concentrations of $0.05,0.5,1,2,3,5,10,20$ and $30 \mu \mathrm{M}$, respectively. CHO cells were treated with equivalent Dox concentrations of $0.01,0.05,0.5,1,2,3,5,15$ and $30 \mu \mathrm{M}$, respectively. The cells were washed twice with PBS and cultured for $48 \mathrm{~h}$. MTS assay was used to determine the cell viability.

\section{In vivo Tumor Inhibition Study}

The animal study was approved by the Ethical Committee of the Institute of Basic Medical Sciences, Chinese Academy of Medical Sciences. All animal procedures were approved by the committee on the Animal Care and Use of the Institute of Basic Medical Sciences, Chinese Academy of Medical Sciences and Peking Union Medical College and performed according to the Institutional Animal Care and Use guidelines. BALB/c mice of 6-8 weeks were purchased from Charles River (Beijing, China). For animal colon cancer model, a suspension of $5 \times 10^{5} \mathrm{CT} 26$ cells in PBS was injected subcutaneously on the back. When tumor diameter reached about $5 \mathrm{~mm}$, mice were randomized to 4 groups. PBS, free Dox $(1.16 \mathrm{mg} / \mathrm{kg})$, Apt-HJ (118 nmol/kg) and Apt-HJ-Dox were administered by intraperitoneal injection every day for 20 days. Tumor size and body weight were measured every one or 2 days. Tumor volume was calculated by length $\times$ width $\times$ width $\times 0.5$.

\section{Statistical Analysis}

Statistical analyses were performed by Prism software (GraphPad 5). The statistical significance was calculated by the $t$-test, which was indicated by the $\mathrm{P}<0.05$. All data are presented as a mean value with its standard deviation indicated (mean $\pm \mathrm{SD})$.

\section{Results}

\section{Preparation and Characterization of Apt-HJ}

The overall design of Apt-HJ is illustrated in Figure 1. The structure consisted of four ssDNA chains; each chain was 66-nt long. All chains were made of 3 components: an AS1411 aptamer at the $3^{\prime}$ end, followed by a 5-nt poly-A linker, and the sequence that made up the Holliday junction at the $5^{\prime}$ end. The four strands were expected to form a cross-shaped nanostructure (Apt-HJ) in a self-assembled way, with each arm of the cross made up by a 17-bp double-stranded structure. To evaluate whether the four ssDNA could indeed form the HJ complex, electrophoresis was conducted. As shown in Figure 2A, after heating up the ssDNA at $95^{\circ} \mathrm{C}$ and a gradual transition to room temperature, Apt-HJ was formed as expected.

For characterization of Apt-HJ, we measured its size and zeta-potential by dynamic light scattering (DLS). As

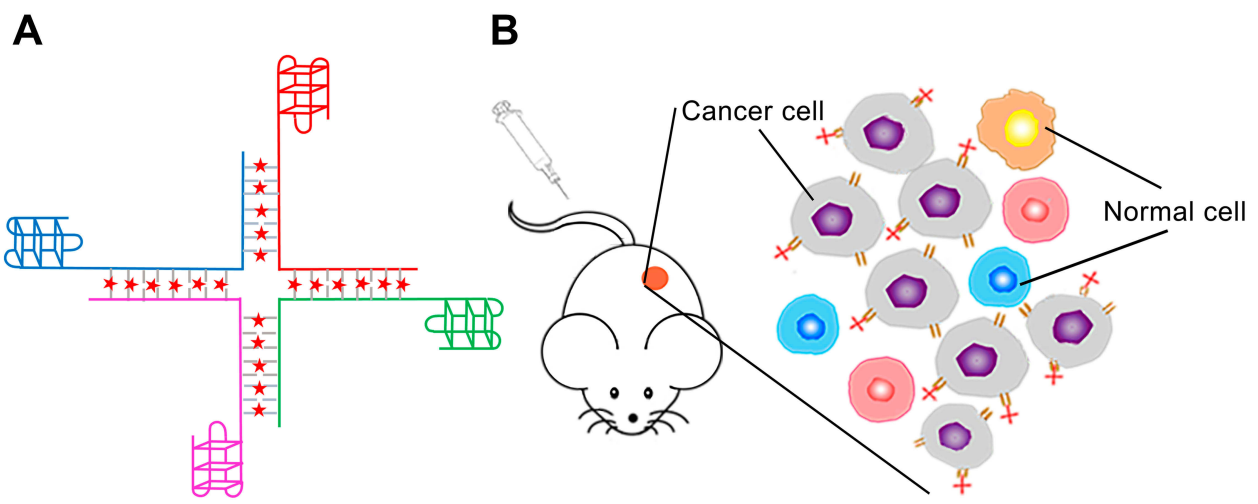

Figure I Schematic illustration of the overall design of Apt-HJ-Dox for targeted delivery of Dox to cancer cells in vivo. (A) The structure of Apt-HJ-Dox, with four single strands assembled into a Holliday Junction through precise base pairing. ASI 4I I was attached to the 3' end of each strand. Dox (red dots) intercalated into the CG pairs of the double-strand DNA. (B) Schematic showing that Apt-HJ-Dox might enter tumor tissue and selectively bind with nucleolin-positive target cancer cells. 

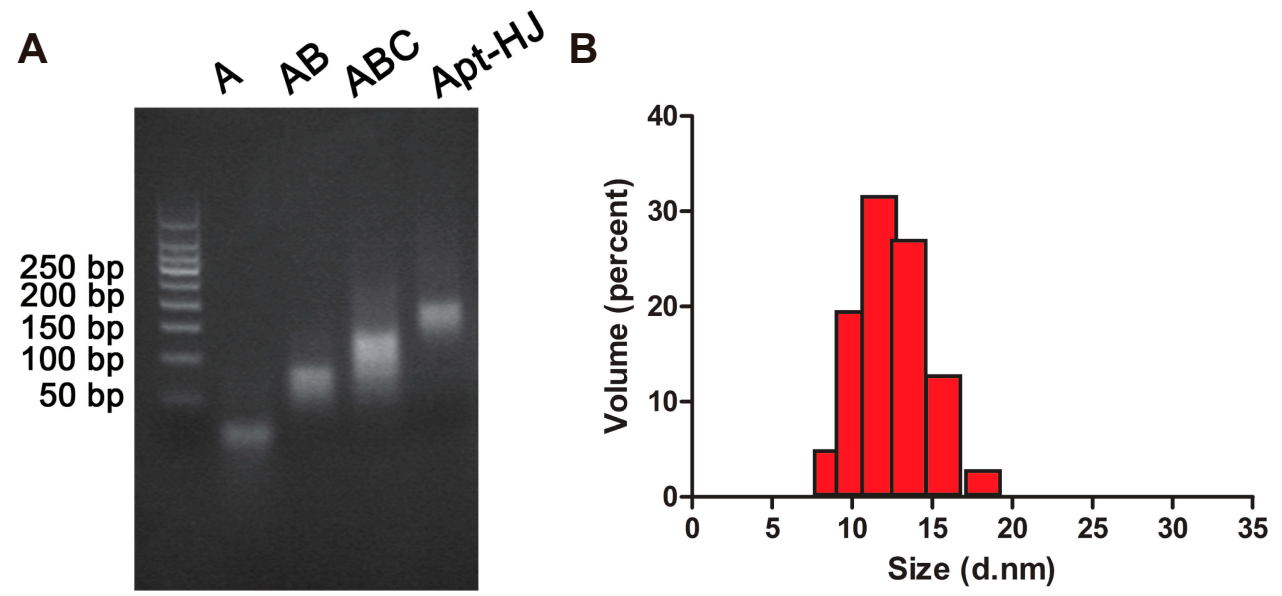

Figure 2 Preparation and characterization of the DNA nanocarrier Apt-HJ. (A) Electrophoresis for verifying the formation of $A p t-H J$. Lanel-3 (A, $A B$, and $A B C)$ represented the complexes formed by one, two, or three DNA single strands. Lane $4(\mathrm{Apt}-\mathrm{HJ})$ represented the complex formed by 4 ssDNA with aptamers attached. (B) Estimation of the particle size of Apt-HJ with dynamic light scattering. Apt-HJ had an average size of $12.45 \pm 2.16 \mathrm{~nm}$.

illustrated in Figure 2B, Apt-HJ had an average size of $12.45 \pm 2.16 \mathrm{~nm}$, and an average zeta-potential of $-11.6 \mathrm{mV}$.

\section{Serum Stability of Apt-HJ}

Durability in serum is a vital prerequisite for nanostructures to serve as drug carriers, because nucleases abound in serum and can digest DNA. Enhancing the resistance to nucleases is thus particularly important for DNA nanostructure to function as SDDS. Exonucleases tend to digest DNA from both ends. For each strand of Apt-HJ, its 3' end is made of the AS1411 aptamer, which is nuclease-resistant due to its G-quadruplex structure. ${ }^{21}$ As a result, the $5^{\prime}$ end of each strand of Apt-HJ needs to be strengthened for nuclease-resistance. Phosphorothioate modification is frequently used to enhance the nuclease resistance of oligonucleotide. $^{23}$ Therefore in this study, to further improve the nuclease-resistance of Apt-HJ, the first nucleotide at 5 '-end of each DNA strand was modified with phosphorothioate. To assess the serum stabilities of phosphorothioate-modified and non-modified Apt-HJs, the two types of Apt-HJs were incubated in $50 \%$ serum for various time periods, and evaluated by electrophoresis. As exhibited in Figure 3, the half digestion time of phosphorothioate-modified Apt-HJ was about $16 \mathrm{~h}$, while that of non-modified Apt-HJ was about $8 \mathrm{hrs}$. The results indicated that phosphorothioate modification improved the nucleases resistance of Apt-HJ, and that the phosphorothioate-modified Apt-HJ had stronger serum stability and may serve as a better drug carrier in vivo compared with non-modified Apt-HJ. Therefore, phosphorothioate-modified Apt-HJ was used in subsequent experiments.

\section{Affinity of Apt-HJ to Target Cancer Cells}

Each Apt-HJ complex had four built-in aptamers and was thus quadrivalent. In order to evaluate whether the aptamers could still bind with the target cell after they were assembled into Apt-HJ, and to compare the target affinity of the quadrivalent Apt-HJ vs. the monovalent free aptamer, flow cytometry study was performed. Each Apt-HJ complex and each free AS1411 aptamer were labeled with a single fluorescent molecule (FAM), to ensure that the fluorescent signals could be compared when equimolar Apt-HJ or AS1411 were used to stain the cells. Next, nucleolin-positive CT26 cells or CHO control cells were incubated with fluorescence-labeled Apt-HJ or AS141, and evaluated by flow cytometry. Multiple prior studies have established that CT26 colon cancer cells overexpress nucleolin in cell membrane, while $\mathrm{CHO}$ cells basically have no nucleolin expression. ${ }^{24-26}$ Cells incubated with FAM-labeled random DNA were used as the control. As shown in Figure 4, in CT26 cells, Apt-HJ generated significantly stronger fluorescent signal than free AS1411 (Figure 4A); while in $\mathrm{CHO}$ cells, both Apt-HJ and free AS1411 generated weak signals (Figure 4B). The results indicated that, compared to the monovalent free AS1411 aptamer, the quadrivalent Apt-HJ markedly strengthened the binding to CT26 target cells.

\section{Drug-Loading Capacity of Apt-HJ}

The anticancer drug doxorubicin (Dox) was loaded into Apt-HJ, making use of the fact that doxorubicin tends to intercalate into dsDNA structures to form a Dox-DNA complex. ${ }^{16}$ Free Dox emits a red fluorescence that will 
A

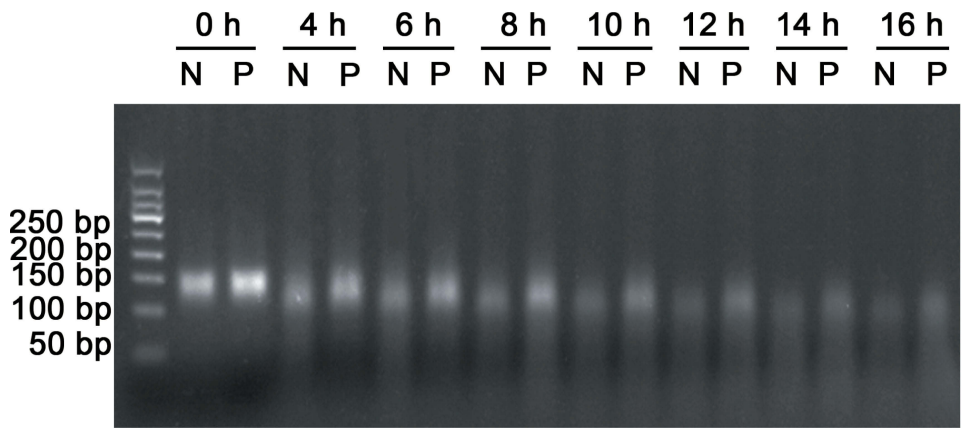

B

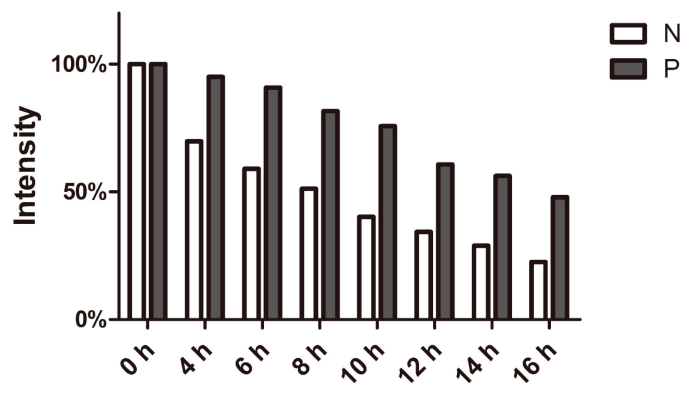

Figure 3 Phosphorothioate-modification enhanced the serum stability of Apt-HJ. (A) Electrophoresis of Apt-HJ complexes that had incubated in $50 \%$ serum for various times (N: non-modified Apt-HJ; P: Phosphorothioate-modified Apt-HJ). (B) Numeric representations of the amounts of DNA complexes. The gray-scale values were obtained by analyzing the gel with Quantity One software.

A

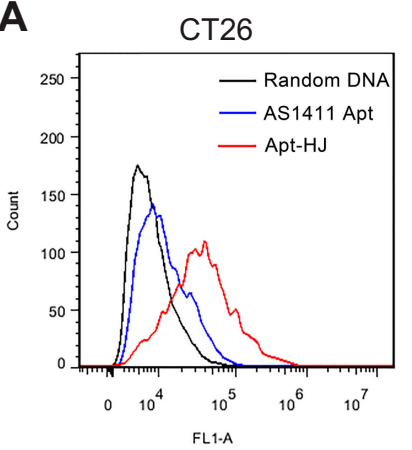

B

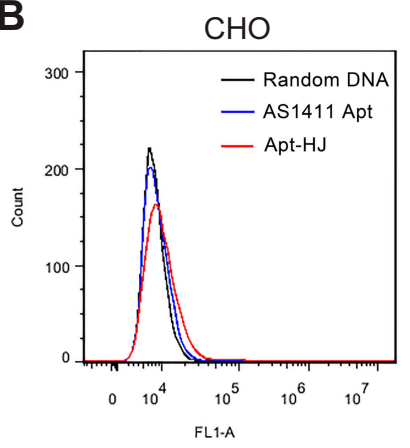

Figure 4 Bindings of Apt-HJ and ASI4II to CT26 (A) or CHO cells (B). Each AptHJ complex or each free ASI4II aptamer was labeled with one FAM. The cells were incubated with FAM-labeled random DNA (black lines), Apt-HJ (red lines), or free ASI4II (blue lines), washed and analyzed by flow cytometry. Cells stained by FAM-labeled random DNA were used as the control.

be quenched when the drug intercalates into dsDNA. This property was utilized to evaluate the drug loading capacity of Apt-HJ. Apt-HJ was mixed with Dox at increasing molar ratios, and mixture's fluorescence spectrum was analyzed. As shown in Figure 5, the fluorescence was almost quenched when the molar ratio of Apt-HJ to Dox increased to 1:17, indicating that most Dox molecules had intercalated into the DNA structure of Apt-HJ under this condition. The results suggested that one Apt-HJ could load approximately 17 Dox molecules when forming the Dox-loaded Apt-HJ complex (Apt-HJ-Dox).

\section{Cellular Uptake of Apt-HJ-Dox}

To evaluate whether Apt-HJ could selectively delivery Dox to target cancer cells, Apt-HJ-Dox or free Dox was incubated with CT26 target cells or CHO control cells, which were evaluated by confocal microscopy. After treatment with Apt-HJ-Dox, red fluorescence could be observed in CT26 cells, but barely in CHO cells

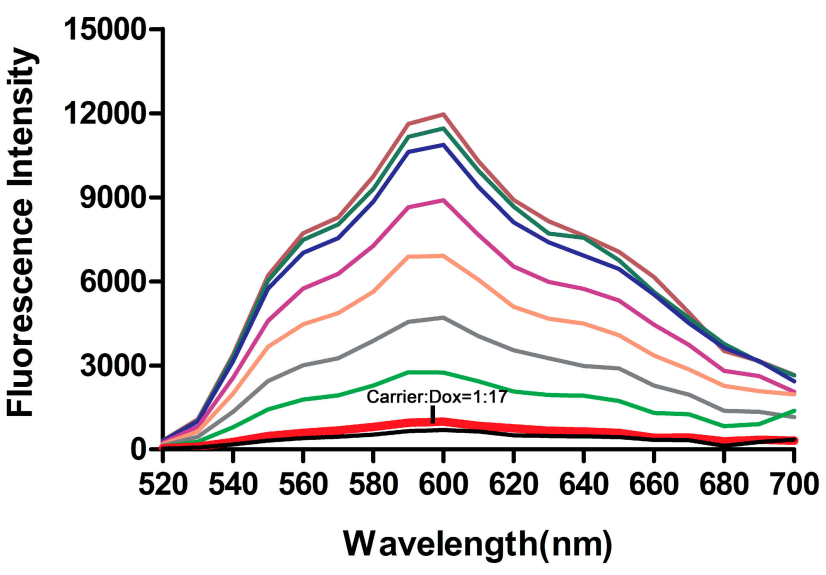

Figure 5 Estimation of the drug-loading capacity of Apt-HJ. Fluorescence spectra of Dox were measured after incubation with increasing molar ratios of Apt-HJ (carrier/Dox ratios from top to bottom: 0, I:5000, I:500, I:100, I:50, I:30, I:20, I:I7, and $I: I)$. $X$-axis represented the wavelength. $Y$-axis represented the fluorescence intensity generated by free Dox molecules that did not intercalate into Apt-HJ. The fluorescence generated by Dox was largely quenched at the carrier/Dox ratio of I:I7 (thick red line pointed by arrow).

(Figure 6), indicating that doxorubicin in Apt-HJ-Dox was delivered into CT26 cancer cells but not the CHO control cells. After treatment with free Dox, however, both CT26 and CHO cells were stained strongly with red fluorescence (Figure 6), indicating that free doxorubicin could not differentiate the two types of cells, and diffused into both.

\section{Apt-HJ-Dox's Cytotoxicity Against CT26 Cancer Cells in vitro}

The above data showed that Apt-HJ-Dox could selectively deliver doxorubicin into CT26 cells. However, it was still necessary to explore whether Apt-HJ-Dox could kill colon cancer cells in a targeted manner. To address this issue, CT26 cancer cells and CHO control cells were incubated 


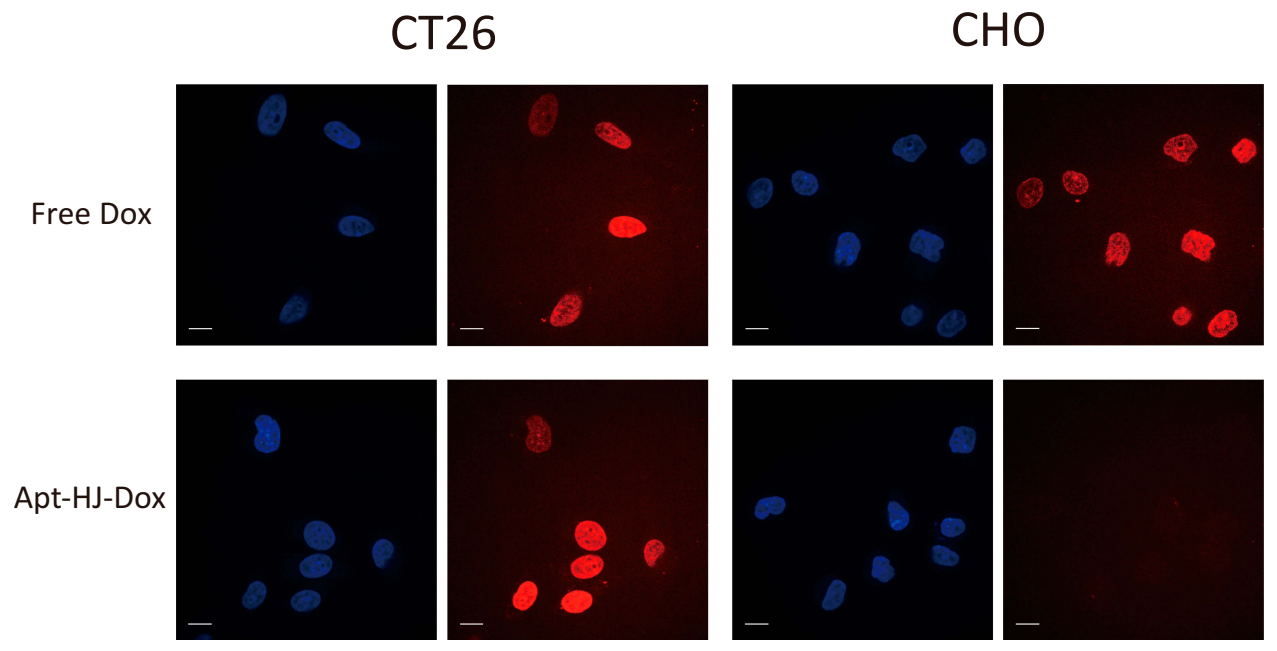

Figure 6 Cellular uptake of doxorubicin by CT26 cancer cells or CHO control cells. The cells were treated with free Dox or Apt-HJ-Dox, and evaluated by confocal microscopy. Scale bar: $10 \mu \mathrm{m}$. The red fluorescence was from doxorubicin. The cell nuclei were stained blue with Hoechst.

with Apt-HJ, free Dox, or Apt-HJ-Dox, and evaluated for cell viability with MTS assay. As shown in Figure 7A, B, free Dox killed both CT26 cells and CHO cells efficiently (cell viabilities: $51.49 \% \pm 4.68 \%$ and $57.23 \% \pm 5.04 \%$, respectively). Apt-HJ-Dox, however, killed CT26 cancer cells efficiently but reduced the damage to $\mathrm{CHO}$ control
A

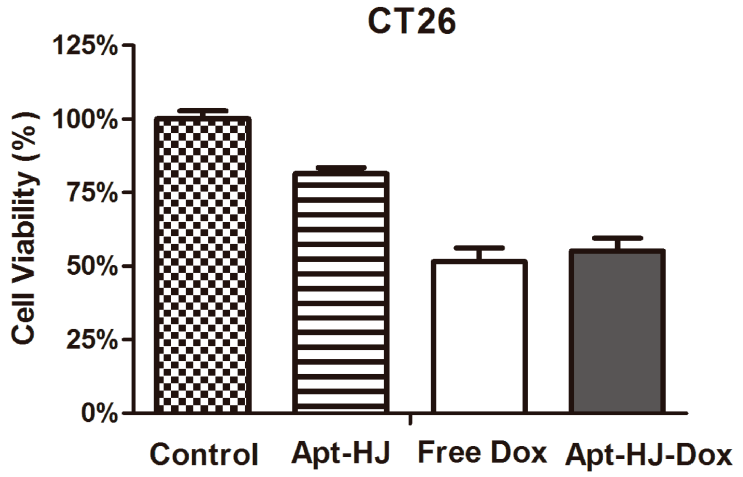

C

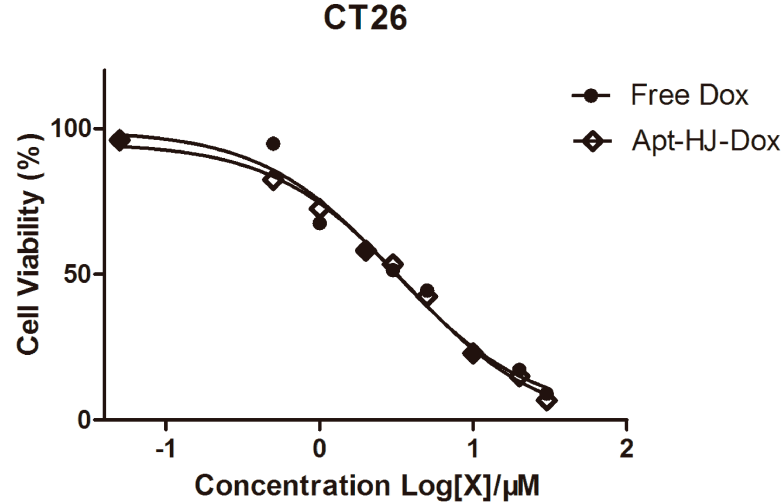

B

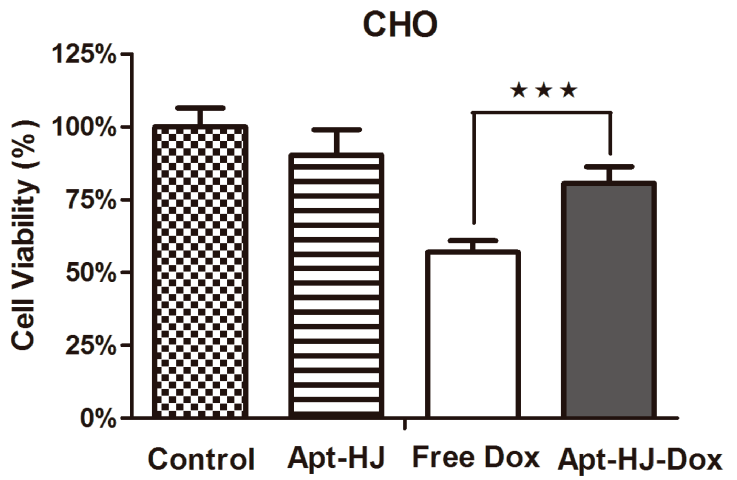

$\mathrm{CHO}$

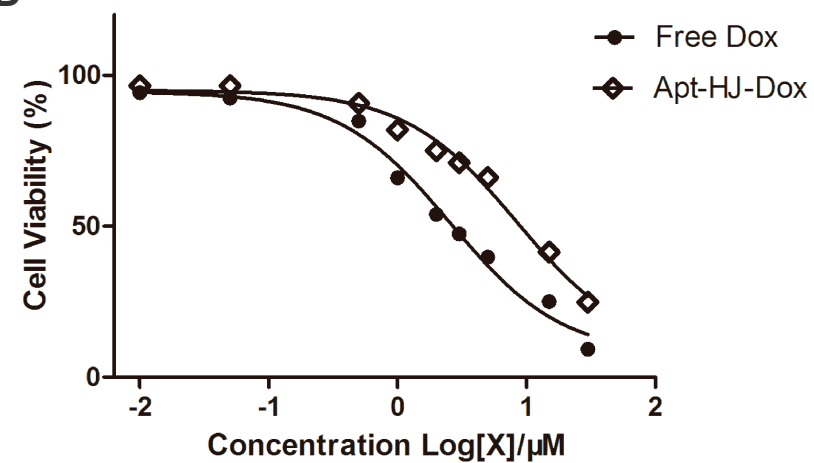

Figure 7 Cytotoxicity generated by Apt-HJ-Dox or free Dox in vitro. Nucleolin-positive CT26 cancer cells (A) or -negative CHO cells (B) were incubated with Apt-HJ, free Dox, or Apt-HJ-Dox for I hr and washed. After 48hrs, cell viability was determined using MTS assay (mean \pm SD, $n=8$ ). Asterisks represent statistically significant difference $(\mathrm{p}<0.05)$. (C) Estimation of IC50s for CT26 cells by Apt-HJ-Dox or free Dox. Nucleolin-positive CT26 cells were incubated with free Dox or Apt-HJ-Dox for I h, at the equivalent Dox concentration of $0.05,0.5,1,2,3,5,10,20$ and $30 \mu \mathrm{M}$, respectively. MTS assay was performed to evaluate the average cell viability ( $\mathrm{n}=8$ ). (D) Estimation of IC50s for $\mathrm{CHO}$ cells by Apt-HJ-Dox or free Dox. Nucleolin-negative $\mathrm{CHO}$ cells were incubated with free Dox or Apt-HJ-Dox for Ihr, at the equivalent Dox concentration of $0.01,0.05,0.5, I, 2,3,5,15$, or $30 \mu \mathrm{M}$, and assayed for average cell viability $(n=8)$. 
cells (cell viabilities: $55.14 \% \pm 4.34 \%$ and $80.72 \% \pm$ $5.71 \%$, respectively). The results indicated that Apt-HJDox killed CT26 cells in a targeted manner. Interestingly, empty vector Apt-HJ also generated mild cytotoxicity to the cells, presumably because of the anti-proliferative activity of AS1411 aptamer. $^{20,21}$ The cell viabilities of CT26 and CHO cells treated with Apt-HJ were $81.39 \% \pm$ $2.07 \%$ and $90.36 \% \pm 8.71 \%$, respectively.

To further characterize the pharmacological property of Apt-HJ-Dox, the IC50 was evaluated. CT26 and CHO cells were treated with Apt-HJ-Dox or free Dox of increasing concentration gradient, and measured for cell viability by MTS assay. As shown in Figure 7C, for CT26 target cells, the IC50s of Apt-HJ-Dox and free Dox were basically similar $(3.660 \mu \mathrm{M}$ vs $3.047 \mu \mathrm{M})$. Nevertheless, for CHO control cells, the IC50 of Apt-HJ-Dox was significantly higher than that of free Dox $(8.481 \mu \mathrm{M}$ vs. 2.579 $\mu \mathrm{M}$ ) (Figure 7D), indicating that Apt-HJ-Dox markedly reduced the damage to control cells. The results further revealed that Apt-HJ-Dox generated a preferential cytotoxicity against the target CT26 colon cancer cells, and mitigated the toxicity to the control cells.

\section{Apt-HJ-Dox Generated Superior Antitumor Efficacy in vivo}

To evaluate the in vivo antitumor efficacy of Apt-HJ-Dox, mice bearing CT26 tumors were systemically treated with PBS, Apt-HJ, free Dox, or Apt-HJ-Dox by daily intraperitoneal injection at a Dox equivalent dose of $1.16 \mathrm{mg} / \mathrm{kg}$ for 20 days. As shown in Figure 8A, Apt-HJ-Dox had the most marked antitumor efficacy, despite that free Dox and Apt-HJ also suppressed tumor growth to certain extents. At the end of the experiment, the average tumor volume of mice treated with Apt-HJ-Dox was five times smaller than that of the control group. Moreover, there was no further weight loss in the Apt-HJ-Dox group vs. the free Dox group (Figure 8B), indicating Apt-HJ-Dox did not generate extra systemic toxicity while enhancing the therapeutic efficacy. The results suggested that Apt-HJ-Dox might have application potential in the development of novel chemotherapy against colon cancer.

\section{Discussion}

Chemotherapy plays an important role in the treatment of advanced metastatic colon cancer, and generally can prolong patient survival. Because of this, several chemotherapies currently serve as the standard treatments for metastatic colon cancer, including FOLFOX (oxaliplatin, 5-Fluorouracil, leucovorin), FOLFIRI (irinotecan, 5-Fluorouracil, leucovorin), CapeOx (capecitabine, oxaliplatin), with or without antibody-based therapeutics (bevacizumab or cetuximab)., Nevertheless, toxicity-related side effects severely limit the long-term application of chemotherapy, and millions of people die of colon cancer each year.

Targeted drug delivery to tumor cells is a promising strategy to reduce the adverse effects. Clinically, FDAapproved targeted drug delivery systems are mainly ADCs, which consist of tumor-targeting antibodies conjugated with drugs. For colon cancer, however, there are no ADCs approved by FDA so far. There are two ADCs (Sacituzumab govitecan, Labetuzumab govitecan) currently undergoing early-stage (Phase I/II) clinic trials for colon cancer treatment, aiming at Trop-2 and CEACAM5, respectively. ${ }^{27,28}$ Therefore, developments are still needed for targeted therapy against colon cancer. In addition to ADCs, nanostructures functionalized with tumor-targeting ligand are also commonly used for the delivery of anticancer

B

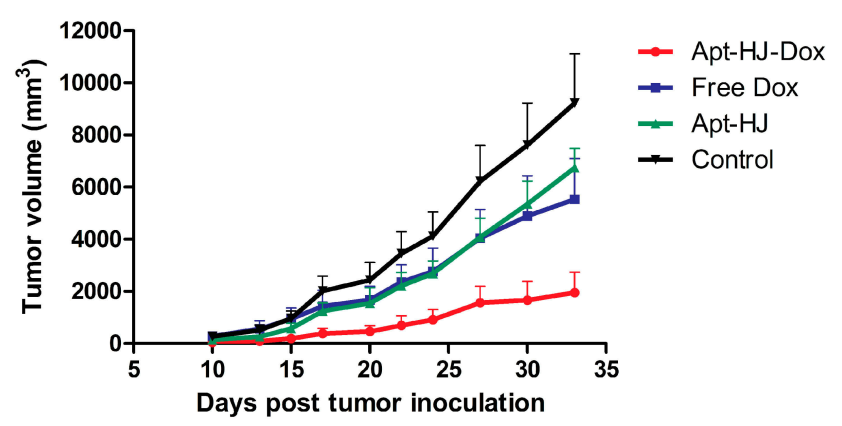

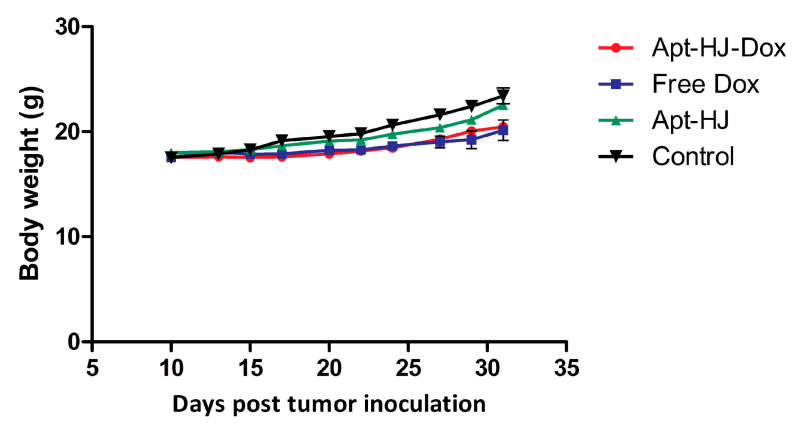

Figure 8 Tumor inhibition by free Dox, Apt-HJ, or Apt-HJ-Dox. Tumor-bearing BALB/c mice were administered with PBS (control), free Dox (I.I6 mg/kg), Apt-HJ (II8 $\mathrm{nmol} / \mathrm{kg}$ ), or Apt-HJ-Dox (with Apt-HJ of II $8 \mathrm{nmol} / \mathrm{kg}$ and Dox of $1.16 \mathrm{mg} / \mathrm{kg}$ ), by daily intraperitoneal injection for 20 days ( $\mathrm{n}=6 \mathrm{mice} / \mathrm{group})$. Tumor volume (A) and body weight (B) were recorded and shown. 
drugs to tumor cells. Among these systems, DNA nanostructures functionalized with aptamers are good candidates for selective drug delivery systems. DNA nanostructures have the advantages of good biocompatibility, negligible immunogenicity, and easy fabrication (usually with a self-assembling process). Furthermore, DNA nanostructures can be readily functionalized by tumor-targeting aptamers.

In this study, aptamer-functionalized $\mathrm{HJ}$ was evaluated for the first time as a drug carrier for targeted treatment of colon cancer. Among DNA structures, HJ is an excellent Dox carrier and has several potential advantages. First, HJ is a nanostructure of relatively small size, with average dimension of about $10 \mathrm{~nm}$. Previous studies have shown that smaller nanoparticles are less likely to be trapped by reticular endothelial system and may infiltrate tumor tissues more efficiently compared with larger nanoparticles. ${ }^{18}$ Therefore, HJ possibly could penetrate tumor and lymph nodes efficiently and improve the utilization of anticancer drugs. Second, the components of HJ can be easily synthesized. Each ssDNA of the HJ used in this study is only 66-nt long (including the AS1411 aptamer, a 5nt spacer, and the HJ part), and can be synthesized easily and economically. Third, HJ has four open ends that can link with tumortargeting ligands to form a quadrivalent nanostructure, which may potentially improve its affinity to tumor cells. Indeed, our results showed that the quadrivalent $\mathrm{HJ}$ had a significantly higher affinity to target cancer cells vs. the monovalent aptamers (Figure 4). Fourth, HJ has relatively high drug loading capacity (Apt-HJ to Dox mol ratio of $1: 17)$ compared to some other DNA nanocarriers, such as three-Way junction-Dox delivery system (1:2.5), AS1411/ FOXM1 aptamers-Dox DNA nanostructure (1:3.5), or PSMA aptamer-Dox conjugate (1.2:1). ${ }^{29-31}$ Fifth, HJ also has certain advantages over some other DNA nanostructures that were used as Dox carriers. DNA nanocentipede and DNA origami are large DNA structures. ${ }^{32,33}$ Because of their size, these carriers tend to be trapped by reticular endothelial systems within the body, and have relatively weak penetration of tumor tissue and lymph nodes. Other DNA structures, such as DNA tetrahedron, though quite small (about $10 \mathrm{~nm}$ ), have difficulty linking with multiple tumor-targeting ligands. ${ }^{34,35}$ Overall, we think that aptamer-modified HJ may serve as an excellent targeted drug delivery system, due to its simplicity, small size, substantial drug-loading capacity, and convenience for linking with multiple tumor-targeting aptamers.

It has long been established that Dox can intercalate into dsDNA. Specifically, the drug tends to intercalate into the CG pairs of double-stranded DNA within the structure, forming the DNA-Dox complex spontaneously. ${ }^{36}$ At present, the detailed drug-DNA interaction driving the process is not entirely clear, but probably involves several types of intermolecular forces, including the Van der Waals force. ${ }^{16}$ The Apt-HJ used in this study had $44 \mathrm{CG}$ pairs in its double-stranded structure. However, owing to the molecular spatial interference, sometimes it is not possible for every CG pair to load a Dox, especially in the case of consecutive CG pairs. ${ }^{37}$ Therefore, the actual drug-loading capacity of a DNA structure is usually lower than the number of CG pairs. With the Apt-HJ used in this study, each DNA complex was estimated to hold approximately 17 Dox molecules (Figure 5).

The mechanisms by which Apt-HJ-Dox preferentially entered CT26 cells rather than $\mathrm{CHO}$ cells (Figures 6 and 7) might involve several aspects. First, DNA normally has difficulty entering the cells, because cell membrane and DNA are both negatively charged and tend to repel each other. Therefore, the negatively charged Apt-HJ-Dox had difficulty entering the CHO control cells. Second, CT26 cells had nucleolin expressed in cell membrane, and could bind with the built-in AS1411 aptamers of Apt-HJ-Dox, thus capturing the DNA-drug complex. The Apt-HJ-Dox attached to CT26 cells subsequently could enter the cells via endocytosis (the detailed process of endocytosis remains unclear at present). After entering the cell, AptHJ was probably degraded by nucleases in lysosomes, and Dox was released intracellularly from the damaged DNA structure. Of note, for CT26 cells, the IC50s generated by Apt-HJ-Dox and free Dox were similar (Figure 7C), indicating that Apt-HJ-Dox and free Dox had similar cytotoxicity against CT26 cells in vitro. This result probably reflected the fact that both free Dox and Apt-HJ-Dox could enter CT26 cells in vitro: Dox entered via simple diffusion, while Apt-HJ-Dox first attached to the cells and then entered via endocytosis. Although CT26 cells took up free Dox and Apt-HJ-Dox in different ways, both could suppress cell growth. As a result, IC50s of Apt-HJ-Dox and free Dox could be similar, if both entered CT26 cells at similar rates in vitro. Importantly, for the $\mathrm{CHO}$ control cells, the IC50 generated by Apt-HJ-Dox was significantly higher vs. free Dox (Figure 7D), indicating that Apt-HJDox reduced the toxicity to the control cells.

Several factors may have contributed to the improved in vivo therapeutic efficacy by Apt-HJ-Dox (Figure 8). First, CT26 colon cancer cells overexpress nucleolin on the cell surface, which can bind with the AS1411 aptamer 
and capture the Apt-HJ-Dox complex, thus increasing the drug delivery to target cancer cells. Second, in order for Apt-HJ-Dox to work in vivo, the DNA structure must resist serum nuclease to maintain integrity. Here in this study, we actually modified the backbone of DNA with Phosphorothioate and significantly improved its serum stability (Figure 3). Third, it has been well established that enhanced permeability and retention (EPR) effect can accumulate small nanoparticles in solid tumor tissue due to leaky tumor vasculature. ${ }^{38}$ Because Apt-HJ is of small size $(12 \mathrm{~nm})$, we hypothesize that passive targeting by EPR may also play a role, allowing the DNA nanostructure to accumulate in tumor tissues. Fourth, owing to aptamer-mediated active targeting, AS1411 aptamer binds to nucleolin in cancer cell membrane, facilitating the uptake of Dox by target cells in vivo. All these mechanisms may have contributed to the improved treatment outcome by Apt-HJ-Dox vs. free doxorubicin. The results indicate that other than ADC, Apt-HJ-Dox may also serve as an alternative strategy for targeted therapy of colon cancer, with the advantage of low production cost, and without the complexity of linker chemistry.

\section{Conclusion}

In conclusion, we designed a novel complex of HJ with four AS1411 aptamers for targeted therapy of colon cancer and showed that such a nanostructure selectively delivers Dox to colon cancer cells in vitro and enhanced the antitumor efficacy in vivo. The results suggest that AS1411modified HJ has the potential for targeted therapy of colon cancer. In the future, AS1411 can be replaced with the aptamers for other tumor markers, and Apt-HJ-Dox may potentially be applied for targeted therapy of other malignancies.

\section{Acknowledgments}

This work was supported by the Ministry of Science and Technology (2017YFA0205504), the CAMS Innovation Fund for Medical Sciences (CIFMS 2016-I2M-3-004), the Natural Science Foundation of China (81572997, 81602712), and the Research Fund to H.Y. from Department of Pathology of PUMC.

\section{Disclosure}

The authors declare that they have no competing interests in this work, and no affiliation to organizations that creates a conflict of interest.

\section{References}

1. Bray F, Ferlay J, Soerjomataram I, Siegel RL, Torre LA, Jemal A. Global cancer statistics 2018: GLOBOCAN estimates of incidence and mortality worldwide for 36 cancers in 185 countries. CA Cancer J Clin. 2018;68(6):394-424. doi:10.3322/caac. 21492

2. Weinberg BA, Marshall JL, Salem ME. The growing challenge of young adults with colorectal cancer. Oncology (Williston Park). 2017;31 (5):381-389.

3. Brody H. Colorectal cancer. Nature. 2015;521(7551):S1. doi:10.1038/ 521S1a

4. Benson AB III, Venook AP, Cederquist L, et al. Colon cancer, version 1.2017, NCCN clinical practice guidelines in oncology. J Natl Compr Canc Netw. 2017;15(3):370-398. doi:10.6004/jncen.2017.0036

5. Van Cutsem E, Cervantes A, Adam R, et al. ESMO consensus guidelines for the management of patients with metastatic colorectal cancer. Ann Oncol. 2016;27(8):1386-1422. doi:10.1093/annonc/mdw235

6. Stathopoulos GP, Koutantos J, Lazaki H, Rigatos SK, Stathopoulos J, Deliconstantinos G. Capecitabine (Xeloda) as monotherapy in advanced breast and colorectal cancer: effectiveness and side-effects. Anticancer Res. 2007;27(3B):1653-1656.

7. Huang Y, Yu Q, Liu Y, et al. Efficacy and safety of chronomodulated chemotherapy for patients with metastatic colorectal cancer: a systematic review and meta-analysis. Asia Pac J Clin Oncol. 2017;13 (2):e171-e178. doi:10.1111/ajco.12456

8. Yu C, Hu Y, Duan J, et al. Novel aptamer-nanoparticle bioconjugates enhances delivery of anticancer drug to MUC1-positive cancer cells in vitro. PLoS One. 2011;6(9):e24077. doi:10.1371/journal.pone.0024077

9. Zhang M, Xu C, Wen L, et al. A hyaluronidase-responsive nanoparticlebased drug delivery system for targeting colon cancer cells. Cancer Res. 2016;76(24):7208-7218. doi:10.1158/0008-5472.CAN-16-1681

10. Masters JC, Nickens DJ, Xuan D, Shazer RL, Amantea M. Clinical toxicity of antibody drug conjugates: a meta-analysis of payloads. Invest New Drugs. 2018;36(1):121-135. doi:10.1007/s10637-017-0520-6

11. Birrer MJ, Moore KN, Betella I, Bates RC. Antibody-drug conjugatebased therapeutics: state of the science. J Natl Cancer Inst. 2019;111 (6):538-549. doi:10.1093/jnci/djz035

12. RobertLyon. Drawing lessons from the clinical development of antibody-drug conjugates. Drug Discov Today Technol. 2018;30:105109. doi:10.1016/j.ddtec.2018.10.001

13. Zhao N, Pei SN, Qi J, et al. Oligonucleotide aptamer-drug conjugates for targeted therapy of acute myeloid leukemia. Biomaterials. 2015;67:42-51. doi:10.1016/j.biomaterials.2015.07.025

14. Stein CA, Castanotto D. FDA-approved oligonucleotide therapies in 2017. Mol Ther. 2017;25(5):1069-1075. doi:10.1016/j.ymthe.2017.03.023

15. Kumar R, Lal S. Synthesis of organic nanoparticles and their applications in drug delivery and food nanotechnology: a review. J Nanomater Mol Nanotechnol. 2014;3:4. doi:10.4172/2324-8777.1000150

16. Agudelo D, Bourassa P, Bérubé G, Tajmir-Riahi HA. Intercalation of antitumor drug doxorubicin and its analogue by DNA duplex: structural features and biological implications. Int J Biol Macromol. 2014;66:144-150. doi:10.1016/j.ijbiomac.2014.02.028

17. Ho PS. Structure of the holliday junction: applications beyond recombination. Biochem Soc Trans. 2017;45(5):1149-1158. doi:10.1042/ BST20170048

18. Qin L, Zhang F, Lu X, et al. Polymeric micelles for enhanced lymphatic drug delivery to treat metastatic tumors. $J$ Control Release. 2013;171(2):133-142. doi:10.1016/j.jconrel.2013.07.005

19. Shao L, Ouchi T, Sakamoto M, Mori S, Kodama T. Activation of latent metastases in the lung after resection of a metastatic lymph node in a lymph node metastasis mouse model. Biochem Biophys Res Commun. 2015;460(3):543-548. doi:10.1016/j.bbrc.2015.03.066

20. Bates PJ, Laber DA, Miller DM, Thomas SD, Trent JO. Discovery and development of the G-rich oligonucleotide AS1411 as a novel treatment for cancer. Exp Mol Pathol. 2009;86(3):151-164. doi:10.1016/j.yexmp.2009.01.004 
21. Bates PJ, Reyes-Reyes EM, Malik MT, Murphy EM, O’Toole MG, Trent JO. G-quadruplex oligonucleotide AS1411 as a cancer-targeting agent: uses and mechanisms. Biochim Biophys Acta Gen Subj. 2017;1861(5 Pt B):1414-1428. doi:10.1016/j.bbagen.2016.12.015

22. Yazdian-Robati R, Bayat P, Oroojalian F, et al. Therapeutic applications of AS1411 aptamer, an update review. Int J Biol Macromol. 2019. doi:10.1016/j.ijbiomac.2019.11.118

23. Eckstein F. Phosphorothioates, essential components of therapeutic oligonucleotides. Nucleic Acid Ther. 2014;24(6):374-387. doi:10.1089/ nat.2014.0506

24. Rangiah K, Tippornwong M, Sangar V, et al. Differential secreted proteome approach in murine model for candidate biomarker discovery in colon cancer. J Proteome Res. 2009;8(11):5153-5164. doi:10.1021/ pr900518v

25. Alibolandi M, Taghdisi SM, Ramezani P, et al. Smart AS1411-aptamer conjugated pegylated PAMAM dendrimer for the superior delivery of camptothecin to colon adenocarcinoma in vitro and in vivo. Int J Pharm. 2017;519(1-2):352-364. doi:10.1016/j.ijpharm.2017.01.044

26. Nejabat M, Mohammadi M, Abnous K, Taghdisi SM, Ramezani M, Alibolandi M. Fabrication of acetylated carboxymethylcellulose coated hollow mesoporous silica hybrid nanoparticles for nucleolin targeted delivery to colon adenocarcinoma. Carbohydr Polym. 2018;197:157-166. doi:10.1016/j.carbpol.2018.05.092

27. Starodub AN, Ocean AJ, Shah MA, et al. First-in-human trial of a novel anti-trop-2 antibody-SN-38 conjugate, sacituzumab govitecan, for the treatment of diverse metastatic solid tumors. Clin Cancer Res. 2015;21(17):3870-3878. doi:10.1158/1078-0432.CCR-14-3321

28. Dotan E, Cohen SJ, Starodub AN, et al. Phase I/II trial of labetuzumab govitecan (anti-CEACAM5/SN-38 antibody-drug conjugate) in patients with refractory or relapsing metastatic colorectal cancer. $J$ Clin Oncol. 2017;35(29):3338-3346. doi:10.1200/JCO.2017.73.9011

29. Taghdisi SM, Danesh NM, Ramezani M, Yazdian-Robati R, Abnous K. A novel AS1411 aptamer-based three-way junction pocket DNA nanostructure loaded with doxorubicin for targeting cancer cells in vitro and in vivo. Mol Pharm. 2018;15(5):1972-1978. doi:10.1021/ acs.molpharmaceut.8b00124
30. Abnous K, Danesh NM, Ramezani M, Charbgoo F, Bahreyni A, Taghdisi SM. Targeted delivery of doxorubicin to cancer cells by a cruciform DNA nanostructure composed of AS1411 and FOXM1 aptamers. Expert Opin Drug Deliv. 2018;15(11):1045-1052. doi:10.1080/ 17425247.2018.1530656

31. Bagalkot V, Farokhzad OC, Langer R, Jon S. An aptamer-doxorubicin physical conjugate as a novel targeted drug-delivery platform. Angew Chem Int Ed Engl. 2006;45(48):8149-8152. doi:10.1002/ (ISSN)1521-3773

32. Li W, Yang X, He L, et al. Self-assembled DNA nanocentipede as multivalent drug carrier for targeted delivery. ACS Appl Mater Interfaces. 2016;8(39):25733-25740. doi:10.1021/acsami.6b0 8210

33. Zeng Y, Liu J, Yang S, Liu W, Xu L, Wang R. Time-lapse live cell imaging to monitor doxorubicin release from DNA origami nanostructures. J Mater Chem B. 2018;6(11):1605-1612. doi:10.1039/ C7TB03223D

34. Dai B, Hu Y, Duan J, Yang XD. Aptamer-guided DNA tetrahedron as a novel targeted drug delivery system for MUC1-expressing breast cancer cells in vitro. Oncotarget. 2016;7(25):38257-38269. doi:10.18632/oncotarget.9431

35. Liu M, Ma W, Li Q, et al. Aptamer-targeted DNA nanostructures with doxorubicin to treat protein tyrosine kinase 7-positive tumours. Cell Prolif. 2019;52(1):e12511. doi:10.1111/cpr.12511

36. Silva EF, Bazoni RF, Ramos EB, Rocha MS. DNA-doxorubicin interaction: new insights and peculiarities. Biopolymers. 2017;107 (3):3. doi:10.1002/bip.22998

37. Lipscomb LA, Peek ME, Zhou FX, et al. Water ring structure at DNA interfaces: hydration and dynamics of DNA-anthracycline complexes. Biochemistry. 1994;33(12):3649-3659. doi:10.1021/ bi00178a023

38. Maeda H. Polymer therapeutics and the EPR effect. J Drug Target. 2017;25(9-10):781-785. doi:10.1080/1061186X.2017.1365878
International Journal of Nanomedicine

\section{Publish your work in this journal}

The International Journal of Nanomedicine is an international, peerreviewed journal focusing on the application of nanotechnology in diagnostics, therapeutics, and drug delivery systems throughout the biomedical field. This journal is indexed on PubMed Central, MedLine, CAS, SciSearch ${ }^{\circledR}$, Current Contents ${ }^{\mathbb{R}} /$ Clinical Medicine,
Journal Citation Reports/Science Edition, EMBase, Scopus and the Elsevier Bibliographic databases. The manuscript management system is completely online and includes a very quick and fair peer-review system, which is all easy to use. Visit http://www.dovepress.com/ testimonials.php to read real quotes from published authors. 